\title{
Breast implant-associated anaplastic large cell lymphoma in an Asian patient: The first case report from Thailand
}

\author{
Peera Thienpaitoon ${ }^{1}$, Wareeporn Disphanurat ${ }^{2}$, Naree Warnnissorn ${ }^{3}$ \\ ${ }^{1}$ PSC Clinic, Nonthaburi; ${ }^{2}$ Division of Dermatology, Department of Medicine and ${ }^{3}$ Department of Pathology and Forensic Medicine, \\ Thammasat University, Pathumthani, Thailand
}

\begin{abstract}
Breast implant-associated anaplastic large cell lymphoma (BIA-ALCL) has received increasing interest among plastic surgeons as a long-term complication of breast augmentation. Although the prognosis is usually good, mortality is a possible outcome. Most of the cases reported in the past two decades have been from the United States, Europe, and Australia, whereas cases of BIA-ALCL in Asia remain rare. Herein, we describe the first known case of BIA-ALCL in Thailand, in which a 32-year-old woman developed BIA-ALCL 3 years after breast augmentation using textured implants. The patient underwent bilateral removal of the implants and ipsilateral total capsulectomy. This case report-the first of its kind from Thailand-should increase awareness of BIA-ALCL among plastic surgeons in Asia. The true incidence of BIA-ALCL in Asia may be underreported.
\end{abstract}

Keywords Breast implant-associated anaplastic large cell lymphoma / Lymphoma, large-cell, anaplastic / Lymphoma, non-Hodgkin / CD30
Correspondence: Peera Thienpaitoon PSC Clinic, 20/263 Prachachuen Road, Pakkred, Nonthaburi 11120, Thailand Tel: +66-2-5744283

Fax: +66-2-5744283

E-mail: peera_au@yahoo.com

Received: January 24, 2020 • Revised: April 8, $2020 \bullet$ Accepted: May 7, 2020

pISSN: 2234-6163 • elSSN: 2234-6171 • https://doi.org/10.5999/aps.2020.00108・ Arch Plast Surg 2020;47:478-482

\section{INTRODUCTION}

Breast implant-associated anaplastic large cell lymphoma (BIAALCL) is a rare variant of T-cell non-Hodgkin lymphoma that was provisionally recognized by the World Health Organization in 2016 [1]. In 2008, de Jong et al. [2] estimated the incidence of BIA-ALCL to be 0.1 to 0.3 per 100,000 women with implants per year. As of July 6, 2019, the US Food and Drug Administration reported 573 pathologically confirmed cases worldwide [3]. The global estimation of risk is increasing, although risk varies across region and according to the implant type. The risk of developing BIA-ALCL is highest for Silimed polyurethane implants (1 in 2,832) followed by Allergan Biocell implants (1 in 3,345) [4]. In recent years, the number of cases reported in the literature has markedly increased, implying that this disease was previously underdiagnosed. BIA-ALCL typically occurs in a delayed fluid collection more than 1 year after implantation around a textured implant that was placed either for reconstruction or cosmetic indications. Cases are most commonly diagnosed during implant revision surgery for delayed seroma [5]. However, patients might have associated symptoms of pain, breast lumps, or asymmetry. All confirmed BIA-ALCL cases are anaplastic lymphoma kinase (ALK)-negative and express the CD30 cell surface protein. The prognosis is favorable, but some cases can be fatal. The mainstay of treatment is complete surgical excision, with possible adjuvant therapy in advanced disease 
$[2,5]$. Only three cases of BIA-ALCL have been reported in Asians, and we present the first report of a BIA-ALCL case in Thailand. The patient provided written informed consent for the publication and use of her images.

\section{CASE}

A healthy 32-year-old woman underwent breast augmentation 3 years ago with an anatomical textured silicone implant (Silimed, Rio de Janeiro, Brazil) placed in the subpectoral plane. She reported swelling in the left breast for 2 weeks. The area of swelling had been growing, without tenderness or fever. She stated that she had not experienced any trauma or accidents in
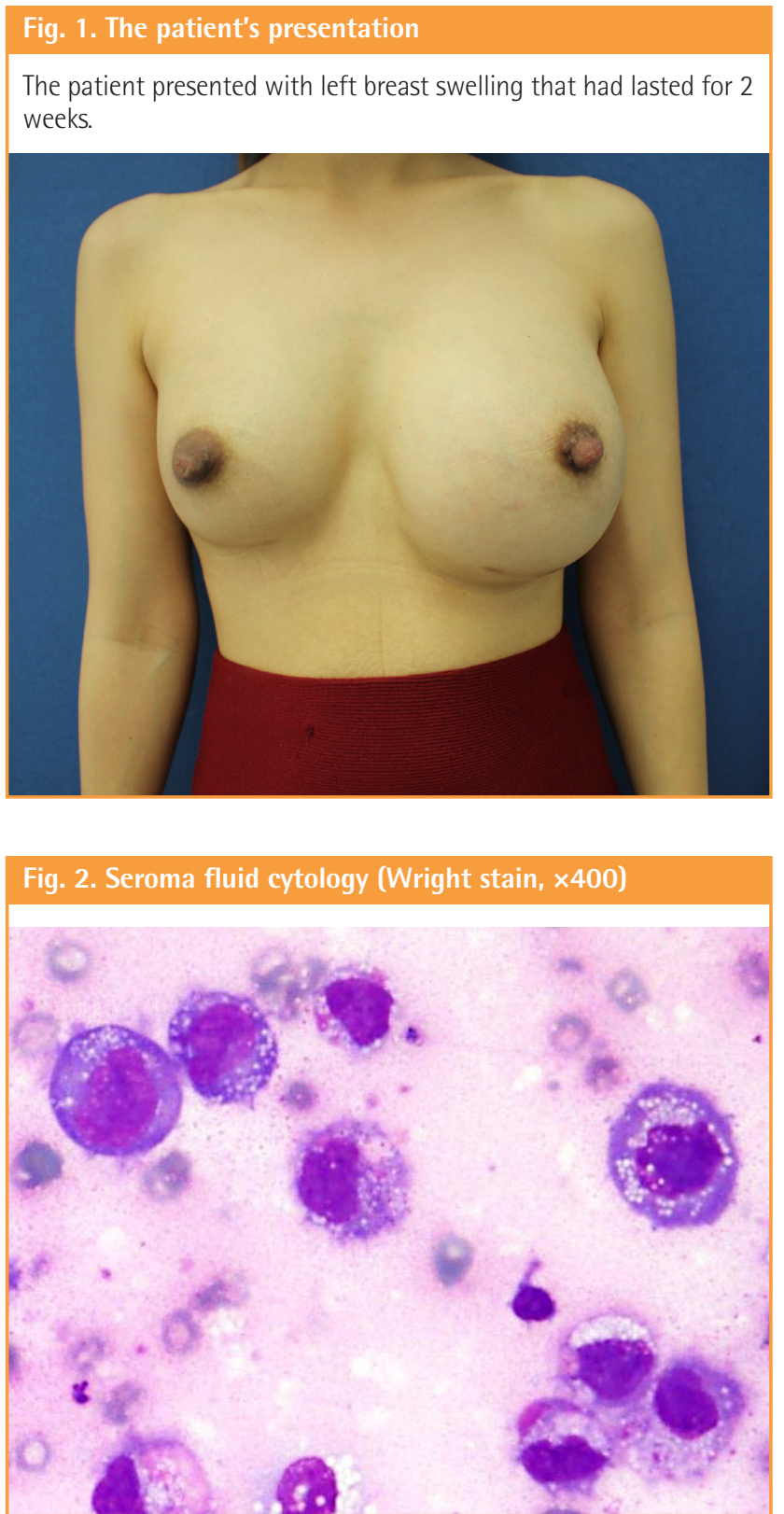

the area of the breast. No adverse events had occurred during the perioperative and recovery periods. Upon examination, asymmetry of the breast was noted, without erythema or ecchymosis (Fig. 1). The axillary lymph node could not be palpated. Hazy, pale-yellow periprosthetic fluid $(150 \mathrm{~mL})$ was drained from the swollen breast and sent for bacterial culture, cytology, and immunohistochemistry. The bacterial culture revealed no growth. Fluid cytology yielded some large atypical mononuclear cells, few histiocytes, and few small lymphoid cells on a proteinaceous background. The large atypical cells had a moderate amount of cytoplasm, intracytoplasmic vacuoles, irregular nuclear shape, lacy hyperchromatic nuclei, and small nucleoli (Fig. 2). Immunohistochemically, the cells were positive for CD30, CD4, CD5, CD43, CD56, TCR-beta F1 (weak), and TIA1, and negative for CD2, CD3, CD7, CD8, CD20, CD68, and ALK (Fig. 3). The diagnosis was BIA-ALCL. A multidisciplinary approach involving collaboration with a radiologist and oncologist

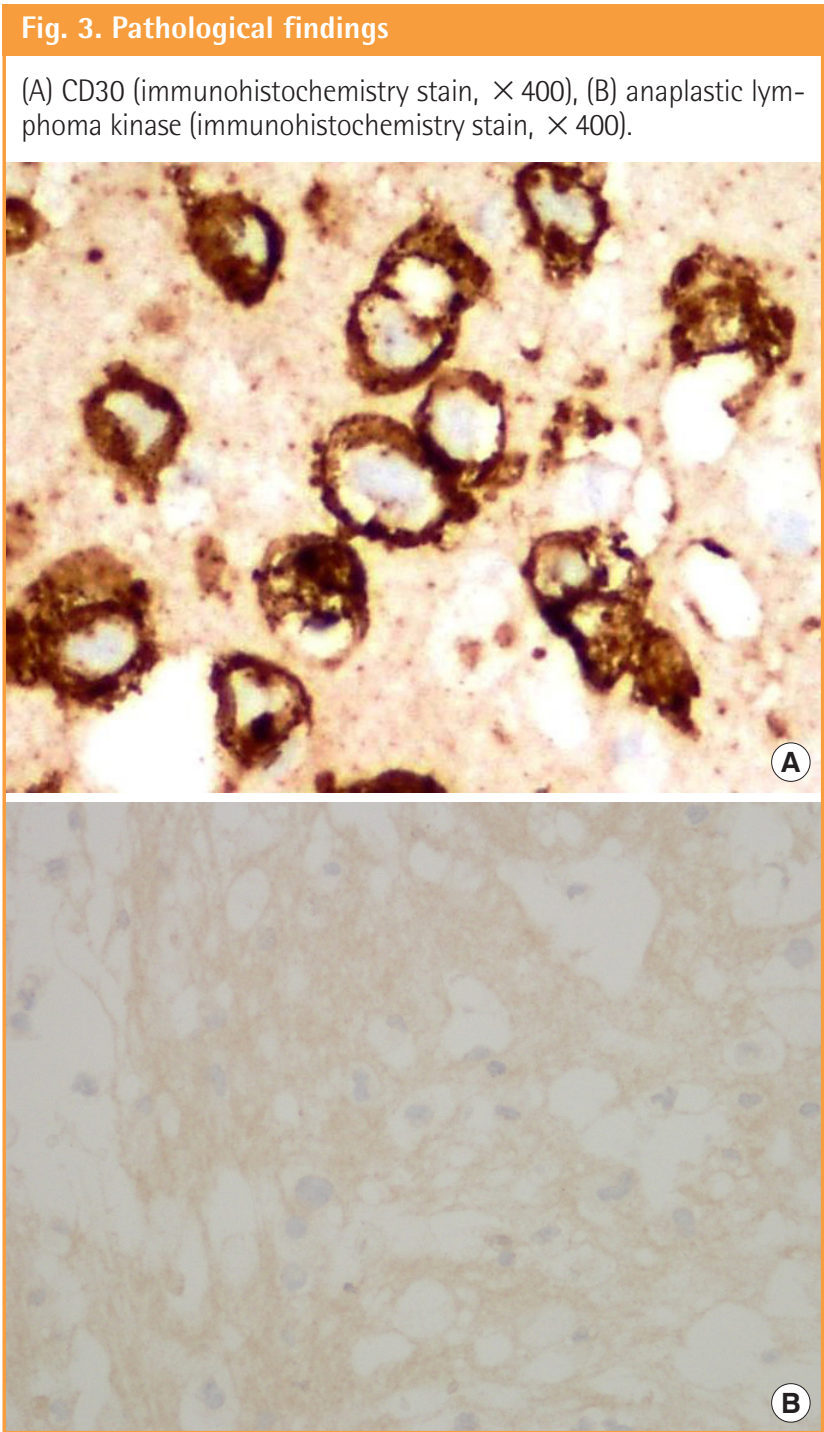


was set up. Magnetic resonance imaging showed no evidence of lymphadenopathy or any suspicious mass at the capsule or inside the breast parenchyma. The surgical approach used an existing inframammary scar. The patient underwent bilateral removal of the implant, ipsilateral total capsulectomy, and removal of yellowish fibrinous material around the implant (Fig. 4). The posterior capsule was injected with saline containing adrenaline in the rib area to facilitate removal. The entire capsule was care-
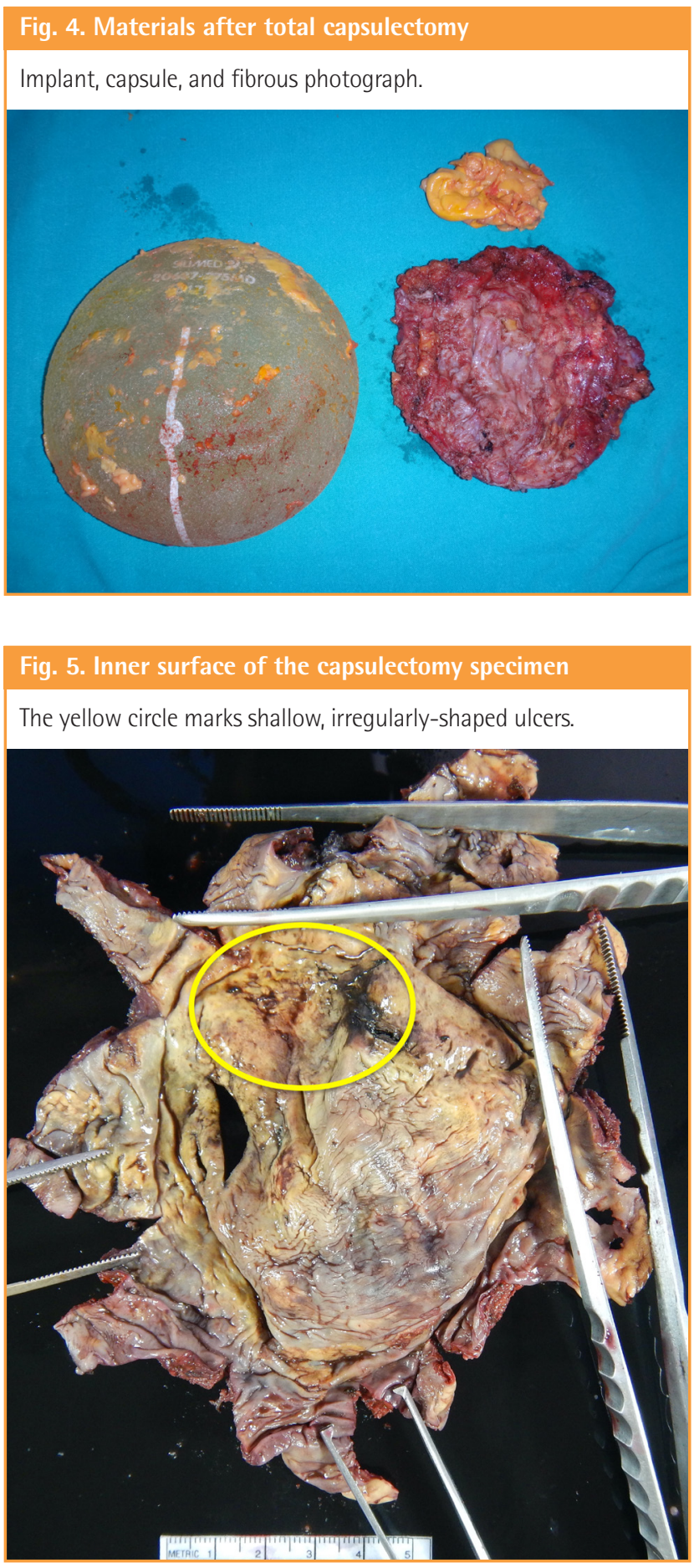

fully evaluated. The inner surface of the formalin-fixed capsulectomy specimen showed two shallow, irregularly-shaped ulcers measuring $1.7 \times 1.5 \times 0.2 \mathrm{~cm}$ and $1.5 \times 0.5 \times 0.2 \mathrm{~cm}$, respectively, that were coated with a thin friable yellow material (Fig. 5). The excised breast capsule was evaluated by hematoxylin and eosin staining. A histological section taken from the ulcer revealed a few large atypical cells with hyperchromatic nuclei embedded in the amorphous eosinophilic proteinaceous material, along with a few histiocytes and small lymphoid cells in the fibrous wall below (Fig. 6A). CD30-positive tumor cells were located within the amorphous material coating the inner surface of the capsule, and no capsular invasion was observed (Fig. 6B). The patient was categorized as having stage $1 \mathrm{~A}$ disease [6]. After complete surgical resection, she continued regular follow-up with her oncologist and plastic surgeon, and has shown no evidence of disease for 2 years.

\section{Fig. 6. Histological section from an ulcer \\ (A) Atypical cells with hyperchromatic nuclei (yellow arrow), and a few small lymphoid cells in the fibrous wall (yellow star) $\left(H \& E_{1} \times\right.$ 200). (B) CD30 (immunohistochemistry staining, $\times 400$ ).}
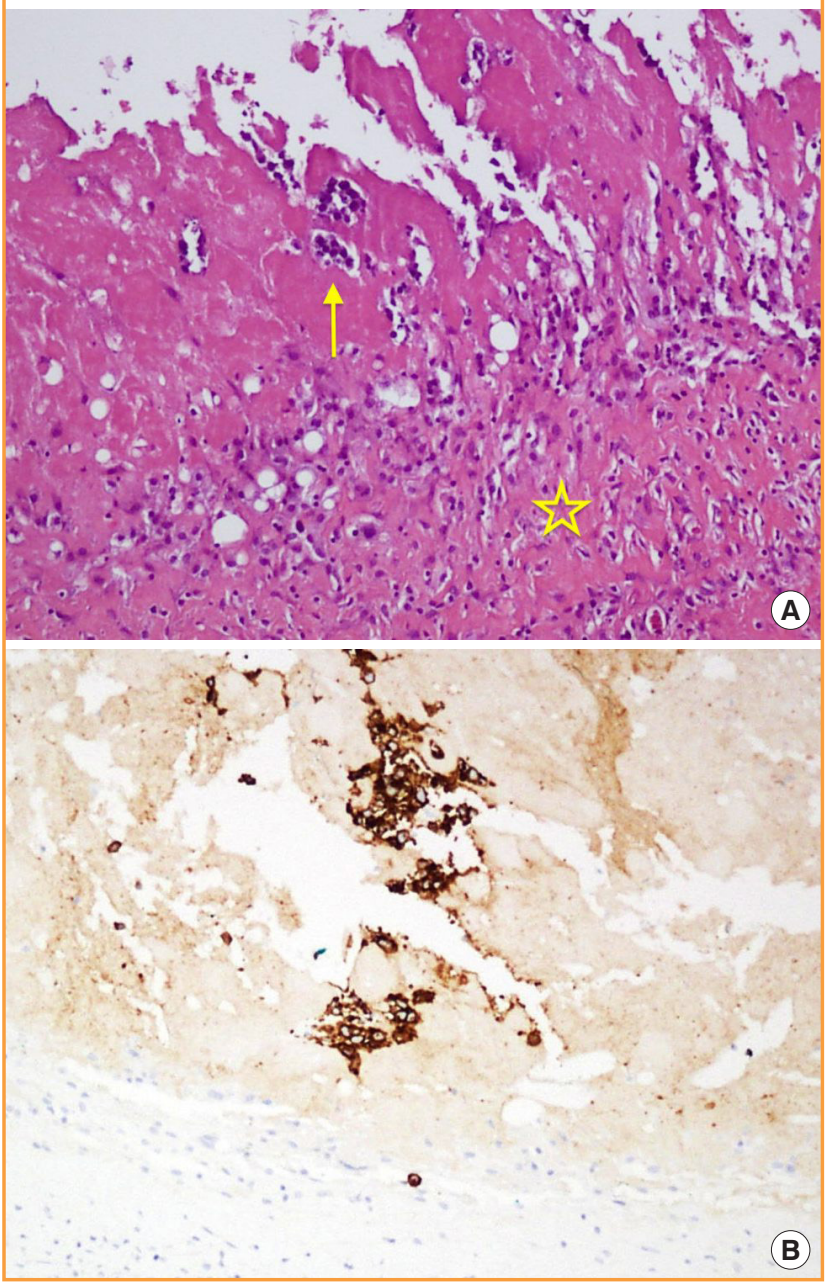


\section{DISCUSSION}

Herein, we described a case of BIA-ALCL that presented with unilateral effusion confined to the capsule of a textured-surface breast implant. To our best knowledge, this is the first case in Thailand and among the first few cases from Asia, which also include a recent patient in Korea and one in Japan [7]. The etiology and pathogenesis of BIA-ALCL have not been clearly established, although proposed causative mechanisms include a chronic $\mathrm{T}$ cell stimulation response to particulate matter in textured implants, the presence of a subclinical biofilm, sequelae of capsular contracture, and genetic predisposition $[8,9]$. The most notable risk factor for BIA-ALCL in our patient was the placement of a textured implant (Silimed) 3 years before the diagnosis. Silimed and Sientra textured devices are manufactured through a process that involves volatilization of ammonium carbonate with heat [10]. Studies have clearly demonstrated that higher-surface-area textured implants are associated with a significantly increased risk of BIA-ALCL $[11,12]$. This may be because larger surface areas make it possible for a larger bacterial load to be present, thereby increasing the risk of lymphocyte stimulation and/or transformation. Hu et al. [13] reported a significant shift towards Gram-negative bacteria in the microbiome in BIA-ALCL in comparison with the Gram-positivedominant microbiome found in capsules without tumors. Another recent controversial study showed no significant differences in the microbiome between BIA-ALCL capsules and normal capsules [14]. Identifying methods to reduce these types of bacteria intraoperatively may reduce the risk of the disease. These methods include betadine pocket irrigation and using the 14-point plan during implant placement to reduce infection. A pathogenic inflammatory immune response in response to the breast implant debris is also under investigation as another possible cause of BIA-ALCL.

Although malignant effusion surrounding an implant is the most common presentation of BIA-ALCL, as in our case, only 3 years passed between implantation and diagnosis for our patient; this interval is markedly shorter than the average (8-10 years) of BIA-ALCL cases [3]. In reported cases of early-onset BIA-ALCL, some patients had a history of implant exchange surgery due to recurrent effusions that did not receive a pathological evaluation until the final diagnosis of BIA-ALCL. In contrast, our patient underwent primary breast augmentation and developed BIA-ALCL 3 years after implantation. She had no history of implant exchange. This underscores the importance of awareness of this disease in patients who present with late seroma around an implant, and regular follow-up can assist in the early diagnosis of BIA-ALCL. However, differences in clinical presentation and the latency between implant placement and disease presentation remain challenges awaiting further research into how genetic predisposition may influence the disease. BIAALCL involves a spectrum of disease. On one hand, it can present as effusion (60\%-85\% of cases), which has an indolent course and a low risk of recurrence after total capsulectomy and implant removal. On the other hand, presentation with a mass or infiltrative-type BIA-ALCL ( $8 \%-18 \%$ of cases) has a more aggressive clinical course, requiring additional therapy $[6,15]$.

We present a confirmed BIA-ALCL case diagnosed at an early stage as malignant effusion contained within the capsule. The patient received surgical treatment (complete capsulectomy and implant removal). Total capsulectomy was challenging in this case because of severe adhesion of the posterior capsule to the rib and intercostal muscles. The injection of tumescent fluid before dissection of the posterior capsule was helpful. Our case report, describing one of the first few known cases in Asia, should alert plastic surgeons to increase their awareness of the disease, and to conduct regular follow-up to facilitate early detection and treatment. Additionally, plastic surgeons should adhere to the guidelines of registries and mandatorily report any cases to determine the true incidence and risk of developing BIA-ALCL.

\section{NOTES}

\section{Conflict of interest}

No potential conflict of interest relevant to this article was reported.

\section{Ethical approval}

The study was exempted from approval by the Institutional Review Board of Thammasat University Hospital (IRB Exemption No. MTU-EC-0-153/63) and performed in accordance with the principles of the Declaration of Helsinki. Written informed consent was obtained.

\section{Patient consent}

The patient provided written informed consent for the publication and the use of her images.

\section{Author contribution}

Conceptualization: P Thienpaitoon. Data curation: P Thienpaitoon. Formal analysis: P Thienpaitoon, N Warnnissorn. Writing - original draft: W Disphanurat, N Warnnissorn. Writing - review \& editing: P Thienpaitoon, W Disphanurat.

\section{ORCID}

Peera Thienpaitoon https://orcid.org/0000-0002-6421-2593 
Wareeporn Disphanurat

https://orcid.org/0000-0001-7843-4915

Naree Warnnissorn https://orcid.org/0000-0002-1902-4366

\section{REFERENCES}

1. Swerdlow SH, Campo E, Pileri SA, et al. The 2016 revision of the World Health Organization classification of lymphoid neoplasms. Blood 2016; 127:2375-90.

2. de Jong D, Vasmel WL, de Boer JP, et al. Anaplastic large-cell lymphoma in women with breast implants. JAMA 2008; 300:2030-5.

3. US Food and Drug Administration. Medical device reports of breast implant-associated anaplastic large cell lymphoma [Internet]. Silver Spring, MD: US Food and Drug Administration; c2019 [cited 2020 May 22]. Available from: https:// www.fda.gov/medical-devices/breast-implants/medicaldevice-reports-breast-implant-associated-anaplastic-largecell-lymphoma.

4. Magnusson M, Beath K, Cooter R, et al. The epidemiology of breast implant-associated anaplastic large cell lymphoma in Australia and New Zealand confirms the highest risk for grade 4 surface breast implants. Plast Reconstr Surg 2019; 143:1285-92.

5. Clemens MW, Nava MB, Rocco N, et al. Understanding rare adverse sequelae of breast implants: anaplastic large-cell lymphoma, late seromas, and double capsules. Gland Surg 2017;6:169-84.

6. Clemens MW, Jacobsen ED, Horwitz SM. 2019 NCCN consensus guidelines on the diagnosis and treatment of breast implant-associated anaplastic large cell lymphoma (BIA-ALCL). Aesthet Surg J 2019;39(Suppl_1):S3-13.

7. Collett DJ, Rakhorst H, Lennox P, et al. Current risk estimate of breast implant-associated anaplastic large cell lym- phoma in textured breast implants. Plast Reconstr Surg 2019;143(3S A Review of Breast Implant-Associated Anaplastic Large Cell Lymphoma):30S-40S.

8. Bizjak M, Selmi C, Praprotnik S, et al. Silicone implants and lymphoma: the role of inflammation. J Autoimmun 2015; 65:64-73.

9. $\mathrm{Hu} \mathrm{H}$, Jacombs A, Vickery K, et al. Chronic biofilm infection in breast implants is associated with an increased T-cell lymphocytic infiltrate: implications for breast implant-associated lymphoma. Plast Reconstr Surg 2015;135:319-29.

10. Stevens WG, Calobrace MB, Alizadeh K, et al. Ten-year core study data for Sientra's Food and Drug Administration-approved round and shaped breast implants with cohesive silicone gel. Plast Reconstr Surg 2018;141(4S Sientra Shaped and Round Cohesive Gel Implants):7S-19S.

11. Jones $\mathrm{P}, \mathrm{Mempin} \mathrm{M}, \mathrm{Hu} \mathrm{H}$, et al. The functional influence of breast implant outer shell morphology on bacterial attachment and growth. Plast Reconstr Surg 2018; 142:837-49.

12. Loch-Wilkinson A, Beath KJ, Knight RJW, et al. Breast implant-associated anaplastic large cell lymphoma in Australia and New Zealand: high-surface-area textured implants are associated with increased risk. Plast Reconstr Surg 2017; 140:645-54.

13. $\mathrm{Hu} \mathrm{H}$, Johani $\mathrm{K}$, Almatroudi A, et al. Bacterial biofilm infection detected in breast implant-associated anaplastic largecell lymphoma. Plast Reconstr Surg 2016;137:1659-69.

14. Walker JN, Hanson BM, Pinkner CL, et al. Insights into the microbiome of breast implants and periprosthetic tissue in breast implant-associated anaplastic large cell lymphoma. Sci Rep 2019;9:10393.

15. Leberfinger AN, Behar BJ, Williams NC, et al. Breast implant-associated anaplastic large cell lymphoma: a systematic review. JAMA Surg 2017;152:1161-8. 